\title{
Actualité documentaire
}

\section{Bernadette Plumelle}

\section{OpenEdition}

\section{Journals}

Édition électronique

URL : http://journals.openedition.org/ries/4077

DOI : $10.4000 /$ ries. 4077

ISSN : 2261-4265

\section{Éditeur}

Centre international d'études pédagogiques

\section{Édition imprimée}

Date de publication : 14 décembre 2014

Pagination : 7-12

ISBN : 978-2-85420-605-0

ISSN : 1254-4590

\section{Référence électronique}

Bernadette Plumelle, «Actualité documentaire », Revue internationale d'éducation de Sèvres [En ligne], 67 | décembre 2014, mis en ligne le 14 décembre 2014, consulté le 22 juin 2020. URL : http:// journals.openedition.org/ries/4077 ; DOI : https://doi.org/10.4000/ries.4077

Ce document a été généré automatiquement le 22 juin 2020.

(c) Tous droits réservés 


\section{Actualité documentaire}

\section{Bernadette Plumelle}

1 CUSSET Pierre-Yves

Les pratiques pédagogiques efficaces : conclusion de recherches récentes

Commissariat général à la stratégie et à la prospective/Paris, août 2014, 42 p. [en ligne]

2 Les études cherchant à évaluer l'efficacité des pratiques pédagogiques, plus nombreuses dans le contexte anglo-saxon qu'en France, se développent. L'auteur rappelle, dans une première partie, les principes et limites des méthodologies mises en œuvre pour évaluer l'efficacité des méthodes pédagogiques. Il présente ensuite des synthèses (méta-analyses) de haut niveau scientifique pour en dégager les résultats. Concernant l'apprentissage de la lecture (déchiffrage et compréhension de texte) et des mathématiques, l'enseignement explicite et l'apprentissage coopératif sont reconnus comme des stratégies d'enseignement efficaces. Pour l'enseignement des sciences, les études disponibles sont moins nombreuses et leurs conclusions encore assez contradictoires. Une dernière partie concerne les études sur les mêmes sujets dans le contexte francophone (Belgique, France). Cependant, les recherches révèlent que les effets des différentes approches sur le niveau des élèves restent relativement modestes au regard d'autres facteurs déterminants qui concernent l'élève lui-même : niveau initial, environnement, capacités. http://goo.gl/nHUOUR

DUBET François (sous la direction de)

Inégalités et justice sociale

La Découverte/Paris, 2014, 304 p.

4 Cet ouvrage collectif, qui s'inscrit dans la continuité d'un colloque (Bordeaux, 2013), cherche à faire le point sur les avancées de la recherche en matière d'inégalités sociales. L'objectif du livre, rappelle François Dubet, est de « répondre à des questions qui structurent l'analyse des inégalités sociales", questions organisées selon quatre axes : comment définir les inégalités ?; les inégalités justes et injustes ; les inégalités et les expériences sociales; les sociologues sont-ils utiles? L'ouvrage réunit les meilleurs spécialistes de la question. Deux contributions portent spécifiquement sur les inégalités scolaires. Marie Duru-Bellat montre combien les outils de mesure retenus par les sociologues partent de présupposés normatifs implicites de nature éthique ou politique 
et conduisent à imposer un modèle de l'égalité des chances. Anne Barrère explique que la dénonciation des inégalités des chances dans le système scolaire a finalement fait oublier le premier objectif de l'école : l'éducation.

DUTERCQ Yves, MAROY Christian (coordinateurs)

Le développement des politiques d'accountability et leur instrumentation dans le domaine de l'éducation : une perspective franco-canadienne

Éducation comparée, 2014, vol. 11, 214 p.

6 Ce dossier, issu de symposiums franco-canadiens, a pour objectif de décrire les instruments de l'action publique en éducation utilisés ces dernières années au Canada et en France (contrat ou convention, projet, évaluation externe, indicateurs, etc.) dans le cadre de politiques recherchant la performance et la reddition de comptes (accountability). Cette notion politique dont la signification s'est étendue et développée parallèlement à l'influence de la doctrine de nouvelle gestion publique (New public management) est discutée dans ce numéro. Le premier article met en évidence la diversité des politiques d'accountability selon les pays; les articles suivants ont pour sujet le pilotage par les résultats en éducation en France et l'usage des statistiques dans le pilotage de l'action publique. Plusieurs études de cas sont proposées ensuite, telles que les politiques de traitement du décrochage scolaire, la lettre de mission des inspecteurs de l'enseignement primaire français dans le cadre d'une nouvelle gouvernance ou la transformation des pratiques individualistes des enseignants dans le cadre de l'accountability en Ontario.

7 FELOUZIS Georges

Les inégalités scolaires

PUF/Paris, 2014, 128 p. (Que sais-je)

8 Les inégalités scolaires se définissent comme un accès différencié aux biens scolaires (filières, diplômes, acquis et compétences...) en fonction de l'origine sociale, du sexe, du parcours migratoire ou de l'origine ethnique. La nature des systèmes éducatifs, leur mode d'organisation et leurs modalités d'enseignement ont un impact fort sur l'ampleur des inégalités d'apprentissage. Quel est le rôle de l'école, quelles sont ses missions? Quels sont les choix en matière de politiques scolaires? Le premier chapitre précise ce que sont les inégalités scolaires et les conceptions de l'égalité. Les chapitres suivants traitent des outils de mesure (données empiriques, indicateurs...) et des analyses empiriques nationales et internationales. Il est question ensuite des causes des inégalités scolaires et des processus de discrimination systématique qui renforcent ces inégalités. Le dernier chapitre est consacré aux politiques de lutte contre les inégalités scolaires, en s'appuyant sur les exemples des États-Unis, de la France et de la Belgique.

KEHM Barbara M., ERKKILÄ Tero (ed.)

Global university rankings. A critical assessment: special issue

European journal of education, mars 2014, vol. $49 \mathrm{n}^{\circ} 1,158$ p. [en ligne]

10 À l'occasion d'une conférence internationale de l'université Jiao Tong de Shanghai pour fêter le $10^{\mathrm{e}}$ anniversaire de son classement, une discussion critique a été lancée sur un certain nombre d'effets indésirables des classements. Certains contributeurs de ce numéro, présents à la conférence, donnent dans leurs articles une évaluation critique et des recommandations sur l'exploitation que l'on peut en faire. Des articles analysent les classements mondiaux d'universités en tant que produits et mesures de la connaissance (méthodologie, fondement théorique, évolutions, etc.). D'autres contributions analysent leurs effets sur les politiques d'enseignement supérieur et les 
pratiques institutionnelles comme, par exemple, les effets des classements sur la diversité et le renouvellement scientifique ou l'intensification de la marchandisation de l'enseignement supérieur au Royaume-Uni. Un dernier article fournit une synthèse des différents points de vue exprimés sur l'impact et les effets indirects non recherchés au niveau national et institutionnel. http://goo.gl/o70aQc

MESKEL-CRESTA Martine, NORDMANN Jean-François, BONGRAND Philippe, et al.

École et mutation : reconfigurations, résistances, émergences

De Boeck supérieur/Louvain-la-Neuve, 2014, 407 p.

L'ouvrage cherche à identifier les mutations subies ou produites par l'école en multipliant et croisant les angles, les domaines et les échelles d'analyse, du plus large (reconfigurations sociétales) au plus fin (expériences et pratiques des acteurs de terrain) et en considérant en retour les effets des pratiques et de leurs transformations aux niveaux pédagogique, didactique, disciplinaire et symbolique. Les contributeurs, spécialistes en sciences sociales et humaines, donnent à voir un espace de réflexion ouvert et interdisciplinaire et entreprennent, au moyen de ce "prisme» de la mutation, de produire une vision renouvelée et intégrée des bouleversements actuels de l'école et de ses reconfigurations.

MORRISSETTE Joëlle, LEGENDRE Marie-Françoise (sous la direction de)

Enseigner et évaluer : regards sur les enjeux éthiques et sociopolitiques

Presses de l'Université Laval/Québec, 2014, 248 p.

Dans un contexte général fortement marqué par l'obligation de résultats, la performance, la compétitivité et l'imputabilité des acteurs, l'évaluation apparaît comme un outil privilégié de gestion des politiques dans les secteurs publics et privés. En réponse à cela, une littérature scientifique s'est développée, qui montre les dérives des évaluations de l'action publique et leurs effets pervers. L'ouvrage rassemble des spécialistes de divers domaines qui réfléchissent à la montée en puissance de l'évaluation comme outil de contrôle et de régulation et soulignent les risques d'instrumentalisation auxquelles peuvent conduire des évaluations chiffrées d'apparence neutre et objective. Les trois premiers textes, d'ordre philosophique et sociopolitique, abordent les dimensions éthiques de l'évaluation à un niveau individuel et collectif.

\section{PÉRIER Pierre}

\section{Professeurs débutants : les épreuves de l'enseignement}

Presses universitaires de France/Paris, 208 p.

Le métier d'enseignant est-il en crise ou définitivement en voie de mutation? Comment peut-on être enseignant? L'auteur, qui a mené plusieurs enquêtes auprès de professeurs débutants dans le secondaire, observe les évolutions en profondeur de ce métier en France. La première partie de l'ouvrage s'intéresse à l'analyse des trajectoires des enseignants débutants depuis leurs origines familiales jusqu'au choix du métier. La deuxième partie a pour objet les rapports des enseignants avec leurs élèves dans la classe, la manière dont ils se construisent et se redéfinissent et le sentiment de "vulnérabilité professionnelle " qu'ils peuvent éprouver. La dernière partie s'attache à identifier les ressources institutionnelles et personnelles mobilisées par l'enseignant pour se forger son métier. Face aux défis pédagogiques à surmonter, les nouveaux enseignants développent un rapport pragmatique à la pratique, au risque d'un certain 
«bricolage ». Chacun forge ainsi son métier, lui donnant une forme plus individualisée, tout en se professionnalisant.

THIBERT Rémi

Une jeunesse fantasmée, des jeunesses ignorées?

Dossier de veille de l'IFÉ, octobre 2014, n 95, 24 p. [en ligne]

On compte environ 95,2 millions de jeunes (15-29 ans) dans l'Union européenne (2012) et la question de la jeunesse fait l'objet de travaux abondants, tant en France qu'en Europe. Ce dossier, qui ne porte pas spécifiquement sur l'école, tente de répondre aux interrogations sur la jeunesse. Qui sont les jeunes d'aujourd'hui ? Quel rôle l'école jouet-elle dans la fabrique de la jeunesse ? Comment les jeunes quittent-ils leur statut pour devenir adulte? Y a-t-il des valeurs propres à la jeunesse ? L'auteur montre, dans une première partie, des approches différentes des politiques publiques selon les pays et les idées reçues sur la jeunesse. Il est question ensuite des valeurs des jeunes, variables selon les pays et les zones géographiques. La dernière partie a pour sujet l'intégration des jeunes dans la société, leur accès à l'autonomie financière et résidentielle (décohabitation) et leur entrée sur le marché du travail. http://goo.gl/nriEFo

19 WELCH Anthony

Higher education in Southeast Asia: blurring borders, changing balance Routledge/Londres, 2013, 195 p.

L'ouvrage analyse l'évolution de l'enseignement supérieur privé en Asie du Sud-Est. Pris entre les impératifs contradictoires d'une demande très forte et de ressources limitées, l'équilibre entre les systèmes d'enseignement supérieur public et privé s'est modifié de manière très sensible. L'auteur propose des études de cas dans cinq pays (Indonésie, Malaisie, Philippines, Thaïlande, Vietnam). Il analyse les enjeux politiques importants de développement de l'enseignement supérieur dans la mondialisation économique et il étudie les pressions de la mondialisation culturelle, en particulier le rôle de la langue anglaise dans l'enseignement. Le rôle de l'enseignement supérieur dans "l'économie de la connaissance », le GATS (accord général sur le commerce des services) et le commerce transfrontalier dans les services éducatifs sont également traités, y compris l'augmentation du nombre de campus délocalisés en Malaisie et au Viet Nam.

\section{Publications d'organisations européennes et internationales}

21 Eurydice : réseau d'information sur l'éducation en Europe

Teachers' and school heads' salaries and allowances in Europe 2013/14

Commission européenne/Bruxelles, 2014, 118 p. [en ligne]

Ce rapport, commandé par la Commission européenne, établit une étude comparative des salaires et des allocations des enseignants et des chefs d'établissement dans le secteur public et privé dans 33 pays européens. L'enquête porte sur les enseignants et les chefs d'établissement du préscolaire au secondaire supérieur. En 2013/2014, les enseignants ont vu leurs salaires augmenter dans 16 pays européens par rapport à l'année précédente. Les hausses sont dues principalement à des réformes salariales et à l'ajustement au coût de la vie. Dans environ la moitié des pays, le pouvoir d'achat des enseignants en 2014 reste en dessous du niveau de 2009. On trouve des informations 
spécifiques sur la progression des salaires par rapport à l'expérience, les modalités particulières au secteur privé, les allocations possibles accordées pour des qualifications supplémentaires. La première partie concerne l'étude comparative; la deuxième partie donne les informations par pays.

Eurydice : réseau d'information sur l'éducation en Europe

Financing schools: mechanisms, methods and criteria in public funding in Europe Eurydice/Bruxelles, 2014, 104 p. [en ligne]

Le financement de l'enseignement scolaire fait l'objet de nombreux débats et il est considéré comme une priorité majeure de la stratégie Europe 2020. Ce rapport sur le financement des écoles en Europe (mécanismes, méthodes et critères) propose un cadre pour comprendre la structure des systèmes de financement et des flux de financement dans l'enseignement primaire et secondaire général. Il explique les flux financiers à travers les différents niveaux administratifs et analyse les critères et les principes de répartition des diverses ressources pour les écoles en Europe. Il couvre 27 des 28 États membres de l'Union européenne ainsi que l'Islande, le Liechtenstein, la Norvège et la Turquie. http://goo.gl/fFHqJ8

OCDE : Organisation de coopération et de développement économiques

\section{Regards sur l'éducation 2014 : les indicateurs de l'OCDE}

OCDE/Paris, septembre 2014, 594 p. [en ligne]

Ce rapport annuel, organisé thématiquement, présente un ensemble d'indicateurs actualisés et comparables sur les systèmes d'enseignement des 34 pays membres de l'OCDE et d'un certain nombre de pays partenaires. Il fournit des données sur la structure, le financement et les performances des systèmes d'éducation. Il est composé de quatre chapitres: les résultats des établissements d'enseignement et l'impact de l'apprentissage; les ressources financières et humaines investies dans l'éducation; l'accès, la participation et la progression au sein des systèmes d'éducation; l'environnement d'apprentissage et l'organisation scolaire. Cette édition 2014 offre de nouveaux indicateurs sur les établissements d'enseignement privés, les conditions requises pour devenir enseignant et l'accès et la participation aux activités de développement professionnel pour les enseignants. http://goo.gl/IP57ok

OCDE : Organisation de coopération et de développement économiques

Résultats du PISA 2012 : les clés de la réussite des établissements d'enseignement : ressources politiques et pratiques. Volume IV OCDE/Paris, 2014, 564 p., stat. [en ligne]

Plus de 510000 élèves dans 65 pays et territoires ont participé à l'évaluation du PISA 2012 (mathématiques comme domaine principal d'évaluation; sciences et compréhension de l'écrit en domaines mineurs). Ce volume a pour objectif de mettre en lumière les facteurs nécessaires à la réussite des élèves. Le premier chapitre analyse les relations entre les résultats et diverses caractéristiques propres aux établissements et aux systèmes d'éducation. Les chapitres suivants examinent les corrélations entre résultats scolaires et modes de sélection des élèves, affectation des ressources, gouvernance des systèmes d'éducation et environnements d'apprentissage en comparant avec les résultats de l'enquête PISA de 2003. Des études de cas sont présentées pour illustrer des réformes politiques lancées dans les pays qui ont amélioré leurs performances aux épreuves PISA. http://goo.gl/ZCHZTi 
29 ISU : Institut de statistique de l'Unesco, Rapport mondial de suivi sur l'EPT

Recherche enseignants formés pour garantir le droit à chaque enfant à l'enseignement primaire

UNESCO/Paris, octobre 2014, 12 p. [en ligne]

Selon les données de l'ISU, les pays auront besoin de quatre millions d'enseignants pour réaliser l'enseignement primaire universel d'ici à 2015. Il s'agira non seulement de remplacer les départs à la retraite ou ceux qui quittent la profession mais il sera nécessaire également de recruter de nouveaux enseignants (1,4 million) pour universaliser l'accès à l'enseignement primaire. Plusieurs projections sont proposées à 2020 ou 2030. La région confrontée aux défis les plus importants dans ce domaine est l'Afrique subsaharienne qui totalise plus de la moitié (63\%) d'enseignants supplémentaires à recruter, suivie des États arabes. Les questions du financement et de la formation des enseignants sont également évoquées. Une infographie (http://goo.gl/ IQr5mJ) sur le site de l'ISU est proposée de même qu'un atlas (http://goo.gl/FR3noC) interactif.

http://goo.gl/rZzo88

\section{AUTEUR}

\section{BERNADETTE PLUMELLE}

Bernadette Plumelle, ingénieure de recherche, est responsable du centre de ressources et d'ingénierie documentaires (CRID) du Centre international d'études pédagogiques (CIEP). 